The Astrophysical Journal, 497:62-66, 1998 April 10

(C) 1998. The American Astronomical Society. All rights reserved. Printed in U.S.A.

\title{
PIXEL LENSING SEARCH FOR BRIGHT MICROLENSING EVENTS AND VARIABLES IN THE GALACTIC BULGE
}

\author{
ANDRew Gould ${ }^{1}$ AND D. L. DePoy \\ Department of Astronomy, Ohio State University, 174 West 18th Avenue, Columbus, OH 43210; gould@astronomy.ohio-state.edu, \\ depoy@astronomy.ohio-state.edu \\ Received 1997 July 10; accepted 1997 November 10
}

\begin{abstract}
We describe a new method to search for gravitational microlensing toward the Galactic bulge that employs a small camera rather than a conventional telescope and probes new regions of parameter space. The small aperture $(\sim 65 \mathrm{~mm})$ permits detection of stellar flux variations corresponding to magnitudes $7 \lesssim I \lesssim 16$, while current searches are restricted by saturation to $I \gtrsim 15$. The large pixel size $\left(\sim 10^{\prime \prime}\right)$ and $\sim(6 \mathrm{deg})^{2}$ field of view allows observation of the entire bulge with a few pointings. With this large pixel size (and with the even larger 30" point-spread function [PSF] that we advocate) most bulge stars are unresolved, so one is in the regime of pixel lensing: microlensing and other forms of stellar variation are detected from the difference of pixel counts in successive images. We identify three principal uses of such a search. First, the observations are analogous to normal pixel lensing observations of the bulge of M31 but are carried out under conditions where the detected events can be followed up in detail. This permits crucial checks on the systematics of the M31 searches. Second, the search gives a complete inventory of bright bulge variables. Third, "extreme microlensing events" (EMEs) can be found in real time. EMEs are events with maximum magnifications $A_{\max } \sim 200$ which, if they were observed intensively from two observatories, could yield the mass, distance, and speed of the gravitational lens. The instrumentation required to carry out the observations is inexpensive. The observations could be made in parallel with existing microlensing searches and/or follow-up observations. The data reduction is much simpler than in ordinary pixel lensing because the PSF can be fixed by the optics and so does not vary with atmospheric conditions.
\end{abstract}

Subject headings: gravitational lensing — instrumentation: photometers — stars: variables: other telescopes

\section{INTRODUCTION}

Pixel lensing is the search for microlensing of unresolved stars by subtracting successive images of the same field. Crotts (1992) and Baillon et al. (1993) suggested this method to probe the halo of M31 for massive compact halo objects (MACHOSs). In fact, it is the only possible method to conduct such a search from the ground because very few M31 stars are resolved. By contrast, microlensing searches in nearer star fields like the Large Magellanic Cloud (LMC) Alcock et al. 1997b, MACHO; Ansari et al. 1996, EROS) and the Galactic bulge (Udalski et al. 1994, OGLE; Alcock et al. 1997a, MACHO; Alard 1997, DUO) have used the classical technique suggested by Paczyński (1986): they perform repeat photometry on individually identified stars. Since these fields contain millions of resolved stars, the classical approach works quite well and has produced important results.

Nevertheless, one could in principle apply pixel lensing techniques to the LMC and Galactic bulge. Melchior et al. (1997) have carried out a pilot pixel lensing study of the LMC using archival EROS data, and Tomaney (1997, private communication) has begun pixel lensing searches of both the bulge and the LMC using archival MACHO data. However, the primary motivation for pixel lensing searches in these resolved star fields has been to make use of the additional sources that lie below the detection threshold, and so to increase the sensitivity of the existing experiments.

\footnotetext{
${ }^{1}$ Alfred P. Sloan Foundation Fellow.
}

Here we propose a radically different type of pixel lensing experiment: use a small camera with large pixels and with deliberately degraded optics to search for microlensing and other forms of stellar variation toward the Galactic bulge. The initial motivation for this proposal is to mimic (and thereby to better understand) pixel lensing searches toward M31. However, such a search would yield important information on the characteristics of the bulge as well.

\section{M31 PIXEL LENSING}

When Crotts (1992; Columbia-VATT) and Baillon et al. (1993; AGAPE) first proposed pixel lensing of M31, the idea was greeted with extreme skepticism even in the microlensing community. The principal worry was that it would be impossible to take proper account of seeing variation, so the image differences would be too noisy to detect stellar variations. This objection is now clearly laid to rest by the beautiful difference images of Tomaney \& Crotts (1997) which show hundreds of variable stars, many with variations at the level of a few percent of the background galaxy surface brightness. AGAPE also has good sensitivity to variable stars although their technique does not yield such striking visual representations (Ansari et al. 1997). Nevertheless, neither group has reported a confirmed microlensing event. Part of the reason is a shortage of telescope time, itself partly engendered by the initial skepticism. There are a number of microlensing candidates (Crotts \& Tomaney 1997), but the baselines are not long enough to rule out the possibility that these are long-period variables. 
However, even when more data are required, confirmation of events toward M31 will remain more difficult than toward the LMC and the Galactic bulge. There are two main reasons for this increased difficulty. First, the signalto-noise $(\mathrm{S} / \mathrm{N})$ ratio is inevitably much lower for the more distant sources in M31. For LMC sources, and especially for Galactic bulge sources, substantial additional confidence in the microlensing interpretation of the events comes from the close fit of the light curves to the simple three-parameter Paczyński (1986) form. A second related problem is that only for the brightest M31 sources is the $\mathrm{S} / \mathrm{N}$ sufficiently high to allow precise measurements of the light curve, but these are just the sources most likely to be intrinsically variable. While most bright variables repeat (and so can be identified given a long enough baseline), there is always the possibility that a new rare class of variable will more closely mimic microlensing. Indeed, MACHO discovered a new such class of bright variable toward the LMC called "bumpers" (Alcock et al. 1996a). These (as well as several other previously known classes of variables) are easily removed from the LMC search catalog simply by excluding the bright stars. Moreover, bright stars in the LMC can always be followed up spectroscopically if they raise any suspicions. The situation is quite different toward M31. Bright stars cannot be eliminated without also removing all the high $\mathrm{S} / \mathrm{N}$ events. Moreover, spectroscopic follow-up is very difficult and in many cases impossible. Hence, the interpretation of M31 individual lensing candidates will always be less secure than those found toward the LMC or the Galactic bulge. As Crotts (1992) has pointed out, microlensing events in M31 can be distinguished statistically from a new class of variables by comparing the event rates on the near and far side. However, it would be far preferable to positively identify or rule out a new class of variables and so gain greater confidence in the microlensing interpretation of individual events.

Both the disk and the bulge of M31 are being monitored for pixel lensing events. In certain respects, the M31 disk fields are similar to those of the LMC and the M31 bulge fields are similar to those of the Milky Way bulge. The M31 disk is like the LMC in that both have significant populations of early-type stars and in that both lines of sight are relatively devoid of known populations of foreground stars. Thus, if more than a trickle of events are detected, these are probably due to a previously unknown population of halo objects. For the LMC events, these would mostly be in the Milky Way halo, while for the M31 disk events, they would be in the M31 halo. By contrast, lensing of Milky Way or M31 bulge stars can be caused by other bulge stars, so one is learning primarily about a known population. Of course, one may learn unexpected things about these "known" populations. Indeed, the "known" population of lenses toward the Galactic bulge is yielding a number of surprises including a higher-than-expected optical depth and a perplexing excess of short events (Udalski et al. 1994; Alcock et al. 1997a; Han \& Gould 1996; Han 1997).

\section{MIMICKING M31 BULGE PIXEL LENSING USING THE GALACTIC BULGE}

Here we propose to mimic (and so learn more about) M31 bulge pixel lensing by artificially degrading the observing conditions toward the Galactic bulge. To zeroth order, the source populations and the lens populations should be similar in the two bulges. If they are not, this itself would be very interesting. One might also think about mimicking M31 disk pixel lensing by degrading the observing conditions toward the LMC.

\subsection{Observational Parameters}

A moderately aggressive program for M31 bulge pixel lensing might obtain $1 \mathrm{hr}$ exposures every clear night on a $1.3 \mathrm{~m}$ telescope with $1^{\prime \prime}$ seeing and 0.3 pixels. A $2048 \times 2048$ CCD would then cover $100 \mathrm{arcmin}^{2}$. To precisely mimic these conditions for observations of the Milky Way bulge (which is 100 times closer) one would want $30^{\prime \prime}$ pixels and a $100^{\prime \prime}$ point-spread function (PSF). A $2048 \times 2048 \mathrm{CCD}$ would cover $300 \mathrm{deg}^{2}$. For a camera with a $65 \mathrm{~mm}$ diameter primary optic, the exposure time should be 2 minutes. More accurately, the differential extinction in the two directions must be taken into account to maintain similar observing conditions. For definiteness, we focus on $I$ band. For M31, $A_{I} \sim 0.15$. For the Milky Way bulge as a whole, the extinction is highly variable. However, there are broad regions of the southern bulge $\left(b \lesssim-2^{\circ}\right)$ for which the extinction is moderately low, $A_{I} \lesssim 1.3$. For convenience, we adopt $A_{I}=$ 1.3. For comparison, we note that the extinction in the "Blanco A region" of Baade's Window is $A_{I}=0.83$ (Gould, Popowski, \& Terndrup 1998; Alcock et al. 1998). The difference, $\Delta A_{I}=1.15$, then implies that the Milky Way observations require an exposure time longer by a factor 2.9 , or 6 minutes.

\subsection{Optimal Parameters}

While the above parameters would closely reproduce the M31 observations, altering them slightly would give substantially more information from the bulge pixel lensing observations without compromising their value as tests of M31 pixel lensing. In particular, by reducing the pixel size and PSF each by a factor $\sim 3$ to $10^{\prime \prime}$ and $30^{\prime \prime}$ respectively, the $\mathrm{S} / \mathrm{N}$ of detected variations would increase by the same factor. In addition, one would reduce confusion between neighboring variable sources. Similarly, one could increase the exposure time (or number of exposures) to obtain better S/N. To simulate M31 pixel lensing, one could then simply convolve the images with a larger PSF and add appropriate noise.

One might then ask: why not go the limit of small pixels and large apertures? In particular, why not use the Galactic bulge microlensing observations themselves, and simply degrade these to the M31 pixel lensing conditions? Unfortunately, the bulge microlensing observations cannot be degraded to mimic M31 observations because they saturate at $I \sim 15$ which corresponds to $I \sim 24 \mathrm{M} 31$, well below the threshold of detection. In principle, one could use the bulge microlensing telescopes and drastically reduce the exposure times to avoid saturation. However, given the requirements of readout and pointing, this would take an inordinate amount of time at the expense of the basic microlensing search. A small camera is the most efficient way to cover a large field of view while avoiding saturation.

We are led to a choice of $10^{\prime \prime}$ pixels by the following considerations. The camera could be mounted behind the secondary of a telescope aleady dedicated to microlensing observations, either the searches themselves or follow-up observations such as are now being carried out by PLANET (Albrow et al. 1996) and GMAN (Alcock et al. 1996b). The observations would then be carried out in 
parallel with the primary microlensing observations. The field of view should then be large enough so that the resulting images overlap and cover most or all of the $(\sim 50$ $\mathrm{deg}^{2}$ ) of interest in the southern bulge. In particular, one expects that the follow-up observations will attempt to monitor $O(20)$ microlensed sources scattered over the southern bulge, so a $\sim 6^{\circ}$ field (corresponding to $10^{\prime \prime}$ pixels and a $2048 \times 2048$ detector) should ensure overlapping coverage of the entire region.

\subsection{Pixelization Noise}

In order to form a smooth mosaic from many individual images, the PSF must be substantially larger than a pixel. This is also an important consideration for reducing noise when taking the difference of successive images. Gould (1996) showed that the ratio of "pixelization noise" induced by finite pixel size to photon noise is given by

$$
\frac{\text { pixel noise }}{\text { photon noise }} \sim \frac{\kappa n_{*}}{500 \sigma^{4}},
$$

where $\sigma$ is the Gaussian width of the PSF in pixels, $\kappa$ is the ratio of the galaxy surface brightness to the total surface brightness (galaxy plus sky), and $n_{*}$ is the number of photons collected from a "fluctuation magnitude star" $\left(M_{I} \sim-1.2\right)$ during an exposure. Much of the bulge is above sky $(\kappa \sim 1)$. If the observations are carried out in parallel with follow-up of giants, then the exposures will be 1 or 2 minutes. For definiteness, we assume 2 minutes. A 30" PSF and $10^{\prime \prime}$ pixels imply $\sigma \sim 1.3$. The ratio in equation (3.1) is then $\sim 0.5$. Hence, if the PSF were substantially smaller, the pixelization noise would dominate the photon noise.

\subsection{Signal-to-Noise Ratio}

Sensitivity is defined slightly differently for pixel lensing experiments than it is for ordinary photometry. In the latter, one measures the total flux from a star $F$ and then converts to a magnitude $I=-2.5 \log F+C$, where $C$ is a constant. In pixel lensing, one detects only $\Delta F$, the difference in flux from a star between two epochs. One converts this to a magnitude using the same formula, $I=-2.5 \log \Delta F+C$. Note that this "difference magnitude" is not the change in the magnitude of the star (which is unknown). Rather, it is the magnitude of an imaginary star the flux of which is the same as the difference of the fluxes between epochs.

Typical bulge fields (e.g., Baade's Window) have a dereddened surface brightness $I \sim 17.6$ mag $\operatorname{arcsec}^{-2}$ (Terndrup 1988). We again assume a typical extinction of $A_{I} \sim 1.3$. In a 2 minute exposure by a $65 \mathrm{~mm}$ diameter camera with $25 \%$ overall efficiency, a $10^{\prime \prime}$ pixel will collect $\sim 1500$ electrons. Hence, commercially available CCDs with readout noise of $\sim 15 e^{-}$will not seriously degrade the $\mathrm{S} / \mathrm{N}$.

The area of the PSF is $\Omega_{\mathrm{psf}} \sim 0.75 \mathrm{arcmin}^{2}$, implying that the (reddened) Galaxy background light in a PSF is equivalent to $I \sim 10.3$, i.e., $\sim 400 e^{-} \mathrm{s}^{-1}$. If the observations are made in parallel with follow-up observations, one may expect that the total exposure time at each point will be about 30 minutes per night. This means that a difference magnitude of $I=15$ would be detectable at the $11 \sigma$ level. This is about 7 times better than the $\mathrm{S} / \mathrm{N}$ for the same fluctuation taking place in M31 and observed for $1 \mathrm{hr}$ with a $1.3 \mathrm{~m}$ telescope in $1^{\prime \prime}$ seeing.
Assuming that the well depth of the pixels is $\sim 100,000$ $e^{-}$, saturation would occur for sources at $I \sim 7$ in a 2 minute exposure. The only bulge variable sources likely to exceed this limit are supernovae.

\subsection{Uniformity of PSF}

In standard pixel lensing, the PSF varies with the atmospheric conditions. Correcting for these variations is challenging (Tomaney \& Crotts 1997; Ansari et al. 1997). By contrast, the $30^{\prime \prime}$ PSF proposed here could be set by the optics and would not vary in time. The problems of forming mosaics and differencing successive images would therefore be substantially reduced. However, in order to take full advantage of this simplification, the PSF must also be uniform over the image. Otherwise, the PSF would not actually be the same on images the centers of which are offset from one another.

\subsection{Optics}

A uniform PSF can be achieved simply by defocusing an appropriate optical design. Experience with reflecting telescopes makes this assertion seem counterintuitive because an out-of-focus telescope has a doughnut-shaped PSF. However, this shape is caused by the fact that the secondary occults the central portion of the mirror, a problem that does not affect lens-based cameras.

For a CCD with $15 \mu \mathrm{m}$ pixels, the the focal length of the system should be roughly $310 \mathrm{~mm}(f / 4.75$ for a $65 \mathrm{~mm}$ entrance aperture). The simplest choise for the optical design would appear to be an achromatic doublet. However, this has off-axis aberrations that are too severe to provide a uniform PSF. That is, by the time the on-axis image is made sufficiently large by defocusing the lens, the off-axis images are very unsymmetric and substantially larger than the on-axis image. A classic Cooke Triplett (see Smith 1990 or Kingslake 1965) provides one good solution. When in focus, it gives excellent ( $<1$ pixel) PSFs over the entire field covered by a $2048 \times 2048$ detector array. Paradoxically, we do not want such small PSFs. However, the residual aberrations are small enough that even after modest defocusing the on-axis and off-axis images are very similar. Other solutions are also possible.

For traditional telescopes, structural and environmental effects often induce changes in the focus that are of the same order as the size of the PSF. For the camera proposed here, such focus changes are negligibly small compared to the PSF, in part because the PSF is substantially larger and in part because the optical system is smaller, lighter, and hence more stable. For example, we estimate that temperature swings as large as $20 \mathrm{C}$ should cause changes in the optical path of roughly $0.14 \mathrm{~mm}$ which would change the PSF size and shape by $<0.1 \%$. Flexure will be $\sim 0.03 \mathrm{~mm}$ between the horizon and zenith, that is, a 5 times smaller effect.

\section{SCIENCE}

\subsection{Comparison with M31 Pixel Lensing}

The $65 \mathrm{~mm}$ pixel lensing data can be used to perform several types of checks on M31 pixel lensing. The data can be degraded to reproduce the characteristics of a series of M31 images by convolving them with a larger PSF and adding noise. They can then be searched for microlensing events using exactly the same algorithms that are used for M31. The resulting lens candidates can be checked in 
several different ways. First, one could examine the undegraded $65 \mathrm{~mm}$ pixel lensing data which, from $\S 3.4$, should have 7 times better $\mathrm{S} / \mathrm{N}$. If the event is detectable at all from the degraded data, it should be very easy to tell whether it is indeed microlensing from the undegraded data. Second, much of the bulge will be covered in much finer detail by the regular microlensing surveys. While the peak of typical pixel lensing events will not be visible in the regular data set because of saturation, there could well be good photometry of the source star away from the event which would give important clues to the nature of the source. Third, from the $65 \mathrm{~mm}$ pixel lensing data alone, or perhaps in combination with the regular microlensing survey data, the precise position of the source could be found which would permit spectroscopic follow-up.

One could also empirically evaluate the pixel lensing efficiency as a function of peak flux variation and effective timescale. After the algorithm had found all of the events that it thought were microlensing in the degraded image series, one could search both the undegraded images and the regular microlensing survey catalogs for all events. The latter are likely to be close to complete (or at least have well understood completeness) for events that could plausibly be detected from the degraded images. This procedure will automatically take account of the effects of variables, both variables mistaken for microlensing events and confusion caused by variables near genuine microlensing events.

\subsection{Bright Bulge Variables}

Pixel lensing observations of the bulge would easily find all variables with difference magnitudes $I<15$. In principle, one could imagine processing the time series to extract much fainter variables. However, the regular microlensing observations are more efficient at finding these fainter variables. They are insensitive to bright variables because of saturation.

A complete catalog of bright variables would be extremely useful for bulge studies. Previous studies cover only patches of parameter space and suffer from small statistics. Plaut (1970) surveyed $42 \mathrm{deg}^{2}$ centered near $(l, b)=\left(0^{\circ},-10^{\circ}\right)$ and found 637 Miras and other longperiod variables. He conducted a similar search at $(l$, $b)=\left(0^{\circ},+12^{\circ}\right)$. Lloyd Evans (1976) carried out an optical search for bright bulge variables. He obtained 22 epochs for three fields, each about $0.33 \mathrm{deg}^{2}$, at $(l, b)=(1.4,-2.6)$, $(0.9,-3.9)$, and $4.2,-5.1)$. He found 57,38 , and 12 Miras, respectively. A subset of these stars were subsequently studied by Glass \& Feast (1982) in the infrared where most of their luminosity is generated. Terzan, Wehlau, \& Wehlau (1986) found 36 variables in $\sim 0.8 \mathrm{deg}^{2}$ at $(l, b)=(3.5,4.0)$ observed at 57 epochs. Whitelock, Feast, \& Catchpole (1991) made 10-12 JHKL observations of 141 bulge sources at $7^{\circ}<|b|<8^{\circ}$ selected on the basis of the their IRAS colors and found 113 to be Miras. Weinberg (1992) studied 3170 sources classified as variable by IRAS and further selected by IRAS colors. He concluded that these stars, thought to be mostly AGB stars, lay in a highly barred distribution, although the effective magnitude limit of the sample allowed him to map out only the near side of the bar.

A complete sample of bright bulge variables would permit study of the following questions. First, what is the three dimensional distributions of Miras? If the bar found by Weinberg (1992) is confirmed, how does it relate to the bars found in the COBE light (Binney, Gerhard, \& Spergel 1997) and in clump giants (Stanek et al. 1997)? These two bars do not agree with each other and neither agrees with the distribution of RR Lyrae stars which show little if any detectable bar (D. Minniti 1997, private communication). What is the relative number of Miras and clump giants as a function of position in the bulge (and hence of metallicity)? This should be a good indicator of the relative lifetimes of these two phases of evolution as a function of metallicity. How does the period-luminosity relation vary with position (and hence metallicity)?

While Miras are probably the largest class of bright variables, others are also potentially interesting. Are there Type II Cepheids in the bulge? To our knowledge, none have been discovered. Type II Cepheids are usually associated with metal-poor populations, so perhaps none should be expected. However, the $r^{-3.5}$ profile of RR Lyrae stars (a metal-poor tracer) continues in toward the Galactic center to at least $500 \mathrm{pc}$ (D. Minniti 1997, private communication), so the metal-poor population may be quite dense close to the center. In addition, many novae will be discovered, and there may be other rarer classes of variables as well.

\subsection{Extreme Microlensing Events}

Gould (1997) pointed out that extreme microlensing events (EMEs) could potentially be used to measure individual masses, distances, and tranverse velocities for up to 30 bulge lensing events per year. EMEs are lensing events of main-sequence stars with maximum magnification $A_{\max } \sim$ 200. Their small impact parameters $\beta \sim 1 / 200$ imply that it is often possible to measure both the "parallax" and the "proper motion" of an EME. The parallax measurement requires simultaneous follow-up observations from two sites separated by about one Earth radius and yields the size of the Einstein ring projected onto the observer plane, $\tilde{r}_{e}$. The proper-motion measurement yields the angular Einstein radius, $\theta_{e}$. These two quantities can be combined with the measured timescale of the event to extract the three physical parameters. For example, the mass is given by $M=\left(c^{2} / 4 G\right) \tilde{r}_{e} \theta_{e}$.

The accuracy of the photometry needed to measure the parallax of an EME is of order 1\% or better which is moderately challenging. However, the most difficult problem is to identify the event before its peak which is a prerequisite for making intensive follow-up observations from two sites. In principle, it is possible to make this identification by comparing two sets of regular bulge microlensing search observations taken on successive nights. Unfortunately, the EME source stars are generally not in the template, so the EME cannot be found by standard techniques. A pixel Lensing search is required. The regular bulge microlensing data and the $65 \mathrm{~mm}$ diameter camera data can play complementary roles in such a search.

Typical events with magnification $A_{\max } \sim 200$ peak at $I_{0} \sim 13.2$. Assuming an extinction $A_{I} \sim 1.3$ and an Einstein crossing time $t_{e} \sim 10$ days, then 1 day before peak the star will be magnified by $A \sim\left(t_{e} / 1\right.$ day $) \sim 10$, and hence have apparent magnitude $I \sim 17.7$. At this brightness, the "new star" would be clearly visible in the regular microlensing search images, provided one knew where to look. The main problem is how to process the $2 \times 10^{9}$ pixels (covering $\sim 50$ $\mathrm{deg}^{2}$ ) to find the new star. Perhaps the simplest approach would be to degrade the PSF to $\sim 5^{\prime \prime}$ so that, as with the 65 $\mathrm{mm}$ observations, the PSF would be independent of atmo- 
spheric conditions. Then one could directly subtract successive images with minimal processing. Assuming 1 minute exposures on a $1 \mathrm{~m}$ telescope, an $I \sim 17.8$ difference star would be detectable at the $16 \sigma$ level. The whole bulge will probably not be observed every night, but even 2 days before peak, the difference star would be half as magnified, $A \sim 5$, and hence detectable at the $8 \sigma$ level. Unfortunately, for every 40 such events $(A \sim 5)$ that are alerted on, only one will become an EME $(A \sim 200) 2$ days later. This is because the number of events reaching at least magnification $A \gg 1$ is inversely proportional to $A$. The $65 \mathrm{~mm}$ observations can play a complementary role of singling out those candidates that are becoming EMEs. At $4 \mathrm{~h}$ before peak the new star will be $I \sim 15.8$, which, according to $\S 3.4$, would be detectable at the $5.5 \sigma$ level. Even for a quarter night, it would be detectable at the $3 \sigma$ level. While this is far too low a significance to choose candidates, it is adequate to choose among the candidates found from the regular survey data taken a night or two previously. These secondary alerts would be rare enough (less than one per night) so that they could be checked directly using the follow-up telescope.

Note that to maximize the effectiveness of this method of finding EMEs, there should be three such cameras in operation, one in South America, one in Africa, and one in Australia.

We thank Tim Axelrod for making the suggestion to use a small camera rather than degrading telescope observations to do bulge pixel lensing. A. G. thanks the Aspen Center for Physics for its hospitality and for providing a most pleasant environment in which some of the work was carried out. Work by A. G. was supported in part by grant AST 94-20746 from the NSF and in part by grant NAG 5-3111 from NASA. Work by D. L. D. was supported in part by grant AST 95-30619 from the NSF.
Alard, C. 1997, A\&A, 321, 424

Albrow, M., et al. 1996, in IAU Symp. 173, Astrophys. Appl. of Grav.

Lensing, ed. C. S. Kochanek \& J. N. Hewitt (Dordrecht: Kluwer), 227

Alcock, C., et al. 1996a, ApJ, 461, 84

.1996b, ApJ, 463, L67

1997a, ApJ, 479, 119

$1997 \mathrm{~b}$, ApJ, 486, 697

. 1998, ApJ, 494, 396

Ansari, R., et al. 1996, A\&A, 314, 94 1997, A\&A, 324, 843

Baillon, P., Bouquet, A., Giraud-Héraud, Y., \& Kaplan, J. 1993, A\&A, 277,1

Binney, J., Gerhard, O., \& Spergel, D. 1997, MNRAS, 288, 365

Crotts, A. P. S. 1992, ApJ, 399, L43

Crotts, A. P. S., \& Tomaney, A. 1996, ApJ, 473, L87

Glass, I. S., \& Feast, M. W. 1982, MNRAS, 198, 199

Gould, A. 1996, ApJ, 470, 201

. 1997, ApJ, 480, 188

\section{REFERENCES}

Gould, A., Popowski, P., \& Terndrup, D. T. 1998, ApJ, 492, 778

Han, C. 1997, ApJ, 484, 555

Han, C., \& Gould, A. 1996a, ApJ, 467, 540

Kingslake, R. 1965, Applied Optics and Optical Engineering (New York: Academic)

Lloyd Evans, T. 1976, MNRAS, 174, 169

Melchior, A.-L. et al. 1997, A\&A, submitted

Paczyński, B. 1986, ApJ, 304, 1

Plaut, L. 1970, A\&A, 8, 71

Smith, W. J. 1990, Modern Optical Engineering (New York: McGraw Hill)

Stanek, K. Z., Udalski, A., Szymański, M., Kaluzny, J., Kubiak, M., Mateo, M., \& Krzeminński, W. 1997, ApJ, 477, 163

Terndrup, D. T. 1988, AJ, 96, 884

Terzan, A., Wehlau, A. F., \& Wehlau, W. H. 1986, AJ, 92, 809

Tomaney, A., \& Crotts, A. P. S. 1996, AJ, 112, 2872

Udalski, A., et al. 1994, Acta Astron., 44, 165

Weinberg, M. D. 1992, ApJ, 384, 81

Whitelock, P., Feast, M., \& Catchpole, R. 1991, MNRAS, 248, 276 